\title{
Extract, Integrate, Compete: Towards Verification Style Reading Comprehension
}

\author{
Chen Zhang, Yuxuan Lai, Yansong Feng*, Dongyan Zhao \\ Wangxuan Institute of Computer Technology, Peking University, China \\ The MOE Key Laboratory of Computational Linguistics, Peking University, China \\ \{zhangch, erutan, fengyansong, zhaody\} @pku.edu.cn
}

\begin{abstract}
In this paper, we present a new verification style reading comprehension dataset named VGaokao from Chinese Language tests of Gaokao. Different from existing efforts, the new dataset is originally designed for native speakers' evaluation, thus requiring more advanced language understanding skills. To address the challenges in VGaokao, we propose a novel Extract-Integrate-Compete approach, which iteratively selects complementary evidence with a novel query updating mechanism and adaptively distills supportive evidence, followed by a pairwise competition to push models to learn the subtle difference among similar text pieces. Experiments show that our methods outperform various baselines on VGaokao with retrieved complementary evidence, while having the merits of efficiency and explainability. Our dataset and code are released for further research ${ }^{1}$.
\end{abstract}

\section{Introduction}

Reading comprehension has been frequently used in various standardized examinations to evaluate one's language understanding skills, where testtakers are expected to read a long article, answer a series of questions, or verify a given statement according to the passage. For example, in the Chinese Language tests of Gaokao (also known as China National College Entrance Examination), approximately half of the reading comprehension questions are in a verification style. As shown in Table 1 (bottom), students are expected to read a passage, and then select from four choices (A D) the best statement that is the most consistent with the passage, or sometimes, contracts the most to the passage.

While the question answering styled tasks have been intensively studied in the NLP community (Rajpurkar et al., 2016; Lai et al., 2017; Yang et al., 2018; Sun et al., 2020), the verification styled

\footnotetext{
${ }^{*}$ Corresponding author.

${ }^{1}$ https://github.com/luciussss /VGaokao
}

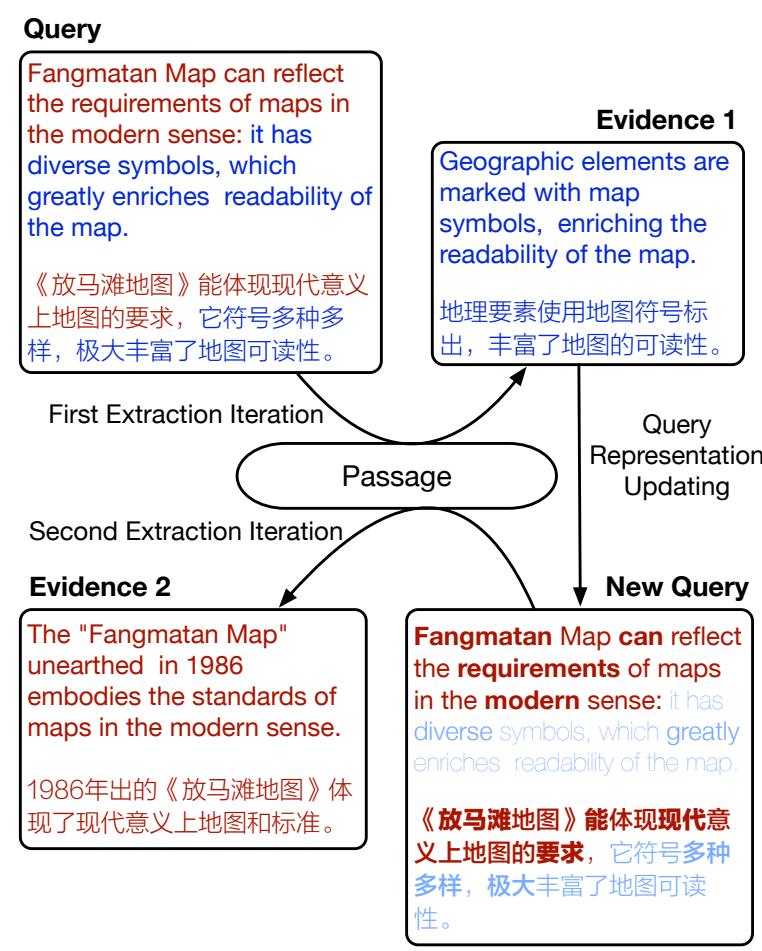

Figure 1: An example of iterative evidence extraction. The darker tokens in bold are more important for the updated query.

MRC task actually receives much less attention. Here, as indicated in the Gaokao Instructions ${ }^{2}$, the abilities of gathering multiple evidence pieces from long articles, distilling supportive evidence, and making decisions accordingly by capturing the subtle difference among similar text pieces (i.e., choices), are necessary skills for Chinese Language learning. This type of questions actually provides an ideal test-bed for natural language understanding research.

In this paper, we present a verification style reading comprehension dataset named VGaokao to highlight the language understanding challenges mentioned above. VGaokao is constructed from the Chinese Language tests of Gaokao. Figure 1

\footnotetext{
${ }^{2}$ http://gaokao.neea.edu.cn/
} 
shows an example in VGaokao, where a statement (one of the 4 choices) should be verified according to the given passage. To do so, we need to extract two evidence sentences from the passage, combine them together to examine how well the statement is supported by the evidence, and finally compare with other choices to arrive at the answer.

As constructed from standardized language test for native speakers, VGaokao involves more language understanding challenges than the datasets constructed from tests for second language learners such as $C^{3}$ (Sun et al., 2020) and RACE (Lai et al., 2017). For example, VGaokao involves more diverse vocabulary and more complex sentence structures. Besides, nearly half of the statements in VGaokao require multiple evidence to verify. Compared with the fact checking tasks such as FEVER (Thorne et al., 2018), where each claim is tagged with a definite label individually, most statements in VGaokao are not absolutely correct nor absolutely incorrect, which requires models to carefully compare one statement with another to choose the most suitable answer according to the given passage.

To address the above challenges in VGaokao, we propose a novel Extract-Integrate-Compete framework, where two query updating strategies, hard masking and soft masking, are designed to iteratively extract multiple complementary evidence for a given statement. Figure 1 shows an example of using soft masking to highlight the tokens whose corresponding evidence has not been found up to current iteration. After evidence extraction, we adaptively filter irrelevant evidence sentences and dynamically determine the number of evidence pieces to be integrated. Options in each question are then verified against retrieved evidence and are compared in a pairwise style to select the most plausible answer.

We empirically investigate the performance of our Extract-Integrate-Compete approach on VGaokao. Experiments shows that our method outperforms end-to-end methods with passage chunking and various evidence selection methods in evidence retrieval F1. Performance gains in evidence selection can further propagate to the final question answering performance.

Our contributions can be summarized as: 1) We propose a novel verification style reading comprehension dataset, VGaokao, which embeds more advanced language understanding skills. 2) We propose a new Extract-Integrate-Compete approach to iteratively select complementary evidence from long articles through a novel query updating mechanism. Our hinge loss based competition component can push the model to capture fine-grained differences among different choices. 3) Experiments show that our approach outperforms a variety of baselines in both evidence retrieval F1 and QA accuracy on VGaokao while showing the merits of efficiency and explainability.

\section{VGaokao: Verification Style Reading Comprehension Dataset}

Standardized language tests have been considered as a test-bed to harvest machine reading comprehension datasets. While most existing efforts focus on SQuAD-like QA datasets (Yang et al., 2018; Kwiatkowski et al., 2019), or cloze style questions (Zhang et al., 2018; Zheng et al., 2019), few efforts are made to verification style questions.

In the Chinese Language test of Gaokao, approximately half of the reading comprehension questions instruct students to select a statement (i.e., an option from four choices) that is the most consistent or contradicting with the given passage. These questions are designed to evaluate students' ability in extracting and integrating information from long passages, and analyzing certain linguistic phenomena or semantic relations among several similar sentences ${ }^{3}$. According to the target language skills, we call these questions verification style questions and convert them into the task of verifying given statements against the articles. Our task is similar in spirit to the fact checking task, FEVER (Thorne et al., 2018). But, unlike the claims tagged with a definite label in FEVER (Supported, Refuted or Not-Enough-Info), the statements in Gaokao questions are designed to be not absolutely correct nor absolutely incorrect. In other words, the options of a question in VGaokao differ in how much they are supported by the passage, which is difficult to be quantified with a value between 0 and 1 . The design of relative correctness is in the purpose of evaluating test-takers' ability to disambiguate very similar options through considering the subtle difference among them.

In this work, we construct a verification style reading comprehension dataset named VGaokao from the Chinese Language test of Gaokao to as-

\footnotetext{
${ }^{3}$ According to the Syllabus of Chinese Language in China by the Ministry of Education.
} 
sess a model's skills of extracting evidence from articles and verifying statements against the retrieved evidence pieces and other options.

\subsection{Dataset Construction}

We construct VGaokao from the Chinese Language examinations in Gaokao and the official mock tests provided by each province. The test questions are designed by the Ministry of Education of China and the examination centers of each province, which are available to the public. We first collect the original set of test article-question pairs and discard the articles and questions that are not of verification style $^{4}$. The remaining articles cover a wide range of topics, including analyzing issues or arguments, introducing recent discoveries in science or engineering, and discussing popular social topics. The remaining questions ask the test-takers to select a statement that is the most consistent with the article or contracts the most with the article, which we can convert into the form of statement verification.

In total, we collect 2,786 passages and 3,512 questions. Each question is paired with 4 options. We randomly sample $80 \%$ of the questions for training and the rest of the questions are used for test.

To quantitatively evaluate the model performance in evidence selection, we randomly sample 25 questions (100 options) from the test set of VGaokao and manually annotate their evidence sentences from the article. On average, there are 1.6 evidence sentences for each option, and the distance between evidence varies from 1 to 9 sentences. This indicates that one has to collect 6.4 evidence on average from the whole passage to answer one question with four options, which makes VGaokao a dataset requiring the ability of gathering and processing multiple evidence.

\subsection{Dataset Analysis}

The basic statistics of VGaokao is shown in Table 2, illustrating challenges from the following aspects.

\section{Advanced Language Comprehension Con-} structed from standardized language tests designed for native speakers, VGaokao requires models to understand more complex passages, compared to other datasets from examinations for second language learners such as $C^{3}$ (Sun et al., 2020).

\footnotetext{
${ }^{4}$ We filter out fictions, proses, and poems from the collection, which focus on evaluating students' ability of aesthetic appreciation. We also discard the questions asking for word meaning explanation or summarizing a certain topic.
}

The main differences can be summarized in three folds. Firstly, in VGaokao, the vocabulary size is 1.6 times larger than that of $\mathrm{C}^{3}$, which brings more diverse words. Secondly, at the sentence level, the average sentence length of VGaokao is 1.6 times longer than that of $\mathrm{C}^{3}$, and the average dependency tree depth of VGaokao is 1.2 times larger than that of $\mathrm{C}^{3}$. Longer sentences and more complicated syntactic structures usually exhibit rich linguistic phenomena, thus requiring models to learn more sophisticated language understanding skills. Lastly, the passages in VGaokao, which contain 1,159 Chinese characters on average, are approximately 3 times longer than that of RACE (Lai et al., 2017) and 10 times longer than that of $C^{3}$ (Sun et al., 2020). The length of most passages even exceeds the maximum input length of general pre-trained language models such as BERT(Devlin et al., 2019). To exploit the long passages, models may need to take discourse structures into consideration so as to better integrate multiple evidence sentences.

As shown in the sample articles and questions from VGaokao and $\mathrm{C}^{3}$ in Table 1, the example article from VGaokao involves domain-specific terminologies such as testosterone and estrogen. The sentences in the VGaokao example are longer and involves more complicated sentence structures such as compound sentences. Besides, the options in VGaokao seem to be more confusing: Option $\mathrm{C}$ and $\mathrm{D}$ both discuss the subtle relationship between the brains and hands, which requires one to carefully discriminate between them based on the passage, while in the $\mathrm{C}^{3}$ example, only Option $\mathrm{B}$ is explicitly mentioned in the passage. These comparisons indicate that VGaokao can be used to evaluate the language comprehension ability at a higher level compared to previous works.

Requirement of Multiple Evidence Longer passages usually require skills of gathering multiple disjoint evidence pieces to verify and compare the candidate statements. As a pilot study, we randomly sample 100 options and manually annotate their golden evidence sentences from their corresponding passages. We find that $47 \%$ of the sampled options require more than one evidence sentences. Furthermore, there are usually certain discourse relationship among multiple evidence pieces required by one statement, such as causality, comparison, or expansion. In the VGaokao example of Table 1, to support Option B, we need to ex- 
$\mathbf{C}^{3}$

…他呆呆地站在那里, 面色㨫崲至极, 双手拧来拧去无处可放。上课前他自以 为成竹在胸, 所以就没带教案和教材。 整整10分钟，教室里鸦雀无声，所有的 学生都好奇地等着这位新来的老师开 口...(共498字)

$\mathbf{Q}$ : 沈从文没拿教材, 是因为他觉得 ( )

A. 讲课内容不多

B. 自己准备得很充分 $\sqrt{ }$

C. 这样可以减轻压力

D. 教材会限制自己的发挥
...He stood there motionlessly, extremely embarrassed. He wrung his hands without knowing where to put them. Before class, he believed that he had had a ready plan to handle different situation so he did not bring his teaching plan and textbook. For up to 10 minutes, the classroom was in perfect silence. All the students were curiously waiting for the new teacher to open his mouth... (498 Chinese characters in total)

Q: Congwen Shen did not bring the textbook because he felt that ( )

A. there were not many teaching contents.

B. he was well prepared. $\sqrt{ }$

C. he could relieve his mental pressure in this way.

D. the textbook was likely to restrict his ability to give a lecture.

\section{VGaokao}

…如果孕妇怀孕期间睪丸素水平较高, 生下的孩子就更容易成为左䊗子，也更 容易在日后得心脏疾病或孤僻症。这也 许可以解释为什么世界上左粠子只占总 人口的 $10 \%$ 。同样, 雌性激素水平较高 的孕妇，所生的女孩食指通常短于无名 指, 而日后患乳腺癌的可能性也较高。 ... (共1126字)

Q: 下列说法符合原文意思的一项是 ( )

A. 在 400 万年进化史中, 人类的手逐渐演

变成使人具有高度智慧的重要器官和大

自然所能创造出的最完美的工具。

B.通常, 左敉子的无名指之所以比食指 长, 是因为孕妇怀孕期间的睪丸素水平 较高。 $\sqrt{ }$

C. 大脑控制手的活动的区域的运动中枢

与语言中枢之间存在着密切的神经联

系，使得手势成为人类沿袭至今的唯一

的肢体语言。

D. 大脑控制手的活动区域，面积达大脑

皮层 $1 / 4$ 。因此大脑皮层特别强烈的兴奋 会使一个简单的手动作顺利实现。
...If pregnant women have higher testosterone levels during pregnancy, their children are more likely to be left-handed and more likely to develop heart disease or autism in the future. This may explain why left-handers only account for $10 \%$ of the total population in the world. Similarly, pregnant women with higher levels of estrogen usually give birth to girls whose index finger is shorter than the ring finger, and they are more likely to develop breast cancer in the future... (1126 Chinese characters in total)

Q: Which statement is most consistent with the passage? ( )

A. In 4-million-year evolutionary history, human hands have gradually evolved into an essential organ enabling human to have high-degree intelligence and the most perfect naturally created tool.

B. In general, the fact that the left-handed ring finger is longer than the index finger is because of the higher testosterone levels during pregnancy. $\sqrt{ }$

C. There is a close neural connection between the motor center and the language center in the area where the brain controls the activity of the hands, making gestures the only body language that has been inherited by humans.

D. The brain controls the active area of hands, which reaches $1 / 4$ of the cerebral cortex. Thus, particularly intense excitement in cerebral cortex makes a simple hand movement smoothly realized.

Table 1: Example articles and questions from $\mathrm{C}^{3}$ (Sun et al., 2020, top) and from VGaokao (bottom).

\begin{tabular}{l|rrr}
\hline & Passage & Question & Option \\
\hline Average Length (Char.) & 1,159 & 18 & 47 \\
Max Length (Char.) & 2,568 & 60 & 147 \\
Vocabulary Size & 96,806 & 1,245 & 34,789 \\
\hline Total Vocabulary Size & \multicolumn{3}{|c}{87,945} \\
\hline
\end{tabular}

Table 2: Statistics of the VGaokao dataset.

tract the first and the third sentences in the sample text as evidence and recognize the causal relationship between them. This observation indicates that more sophisticated skills such as discourse analysis may be helpful for exploiting the evidence pieces.

\section{Our Extract-Integrate-Compete Approach}

We propose a novel Extract-Integrate-Compete approach to address the challenges in VGaokao. As illustrated in Figure 2, our approach includes three stages, iterative evidence extraction, adaptive evi- dence integration and pairwise option competition.

\subsection{Iterative Evidence Extraction}

Generally, in evidence sentence extraction, models are to extract a subset of evidence sentences $\left\{s_{1}, s_{2}, \cdots, s_{n}\right\}$ according to query $q$. One can use an encoder (e.g., averaging over the presentation of each word in the embedding space) to embed query $q$ and evidence candidate $s_{i}$ into dense vectors $q$ and $s_{i}$, and then use a similarity function $\operatorname{sim}(\cdot)$ (e.g., cosine similarity) to obtain the relevance score $f$, i.e., $f\left(q, s_{i}\right)=\operatorname{sim}\left(\boldsymbol{q}, \boldsymbol{s}_{\boldsymbol{i}}\right)$. This method treats each candidate evidence independently, and produces a ranking list. However, when a query requires multiple evidence sentences, independently selecting top $k$ ranked sentences may ignore the complementary relationship between evidence sentences, thus producing inferior results.

In this work, we propose an iterative way to collect multiple evidence for a given query, where the query representation is updated with newly- 


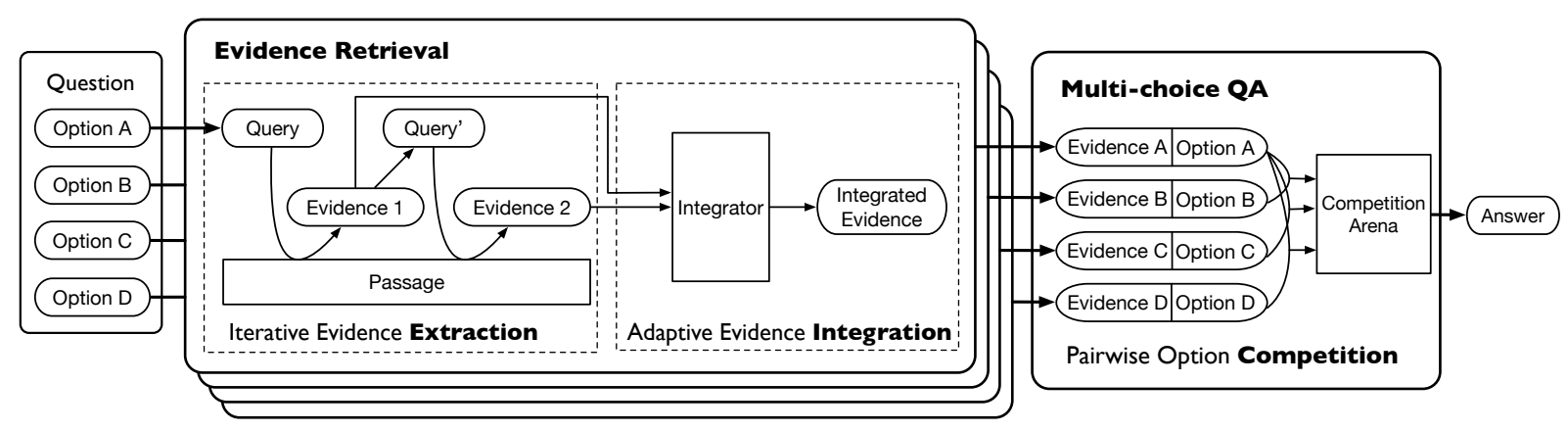

Figure 2: An illustration of our proposed Extract-Integrate-Compete approach.

retrieved information at each extraction iteration. To emphasize the complementary relation between the evidence sentences while avoiding too much overlap, we propose to use masking strategy to reduce the relevance between queries and their retrieved evidence pieces.

Specifically, we assume $e^{t}=\left\{c_{1}^{t}, c_{2}^{t}, \cdots, c_{m}^{t}\right\}$ is the evidence sentence retrieved at the the $t$-th step, $c_{j}^{t}$ is the $j$-th token in $e^{t}$. To search for complementary evidence pieces, we propose to reduce the influence of some parts in $q$ that have been covered by the retrieved evidence sentence $e_{t}$ so that the query representation $\boldsymbol{q}_{t+1}$ for the next step will pay more attention to the parts whose corresponding evidence has not been found.

We design two masking strategies for iterative evidence extraction, i.e., Hard Masking and Soft Masking.

Hard Masking After the $t$-th iteration, we just discard the query tokens that exactly appear in the extracted evidence $e^{t}$. Formally, in the query representation $\boldsymbol{q}_{\boldsymbol{t + 1}}$ for $(t+1)$-th step, the weight $\beta_{i}^{t}$ of the $i$-th query token $q_{i}$ is:

$$
\begin{gathered}
\beta_{i}^{t+1}=\frac{\alpha_{i}^{t+1}}{\sum_{k} \alpha_{k}^{t+1}} \\
\alpha_{i}^{t+1}=\left\{\begin{array}{cc}
0, & q_{i} \in e^{t} \\
\alpha_{i}^{t}, & q_{i} \notin e^{t}
\end{array}\right.
\end{gathered}
$$

Afterwards, we compose the new query representation $\boldsymbol{q}_{\boldsymbol{t}+\mathbf{1}}$ for the $(t+1)$ step by performing a weighted sum of the query token embeddings:

$$
\boldsymbol{q}^{\boldsymbol{t + 1}}=\sum_{i} \beta_{i}^{t+1} \boldsymbol{q}_{\boldsymbol{i}}
$$

In this way, the new query representation is restricted to focus on the unmatched tokens.

Soft Masking Instead of directly assigning zero weights in the hard masking method, soft masking strategy reduces the weights of the already-addressed tokens in the next-step representation. The weight of the $i$-th query token $q_{i}$ is inversely correlated to its matching score to its most similar token in the retrieved evidence set. In practice, we use the dot products of token embeddings to measure the relevance between tokens. Then we calculate the weight $\beta_{i}^{t+1}$ for the $i$-th query token at the $(t+1)$-th retrieval step by adding a negative sign to its highest similarity score and applying a softmax over all the query token weights:

$$
\begin{gathered}
\beta_{i}^{t+1}=\frac{e^{\lambda \alpha_{i}^{t+1}}}{\sum_{k} e^{\lambda \alpha_{k}^{t+1}}} \\
\alpha_{i}^{t+1}=-\max \left(\max _{j}\left(\boldsymbol{q}_{\boldsymbol{i}} \cdot \boldsymbol{c}_{\boldsymbol{j}}^{\boldsymbol{t}}\right),-\alpha_{i}^{t}\right)
\end{gathered}
$$

where $\lambda$ is used to adjust the extent to which we want to widen the weight gap between the matched tokens and the unmatched tokens.

Afterwards, we can obtain the new query representation $\boldsymbol{q}_{t+1}$ similarly to Eq.3.

\subsection{Adaptive Evidence Integration}

In practice, different queries require various amount of evidence pieces. Fixing the numbers of evidence sentences may introduce noise for queries requiring fewer or more evidence sentences. To alleviate this problem, we introduce an evidence integration module to adaptively determine how many complementary evidence pieces are needed for each query.

Specifically, after $t$ steps of beam search, we obtain several evidence chains consisting of $t$ different evidence sentences. In each chain, we reorder and concatenate the evidence sentences according to their order in the original passages, in the hope to maintain the potential discourse relationship between evidence sentences. Then, we feed the integrated evidence chains obtained from different 
numbers of retrieval steps into a reranker, further comparing their semantic similarity to the query. The highest scored evidence chain will be selected as the final evidence chain of the query. This evidence integrator measures the candidate evidence chains as a whole and adaptively filters irrelevant evidence pieces introduced in later iterations.

\subsection{Pairwise Option Competition}

As mentioned in Section 2, we need to carefully discriminate among several options to arrive at the final answer. We thus introduce a pairwise option competition component to the option selection step. We adopt a pre-trained language model $g(\cdot)$ to calculate how the statement $d$ is supported by a retrieved evidence set $c$. During training, for a question that requires choosing the option that is most consistent with the passage, we have a correct option $d^{+}$and several incorrect options $d_{1}^{-}, d_{2}^{-}, \ldots, d_{k}^{-}$, paired with their retrieved evidence sets $c^{+}, c_{1}^{-}, c_{2}^{-}, \ldots, c_{k}^{-}$. We calculate a hinge loss for the pairwise option competition:

$$
\begin{aligned}
& L\left(d^{+}, d_{1}^{-}, d_{2}^{-}, \ldots, d_{k}^{-}\right)= \\
& \sum_{i=1}^{k} \max \left(0,-g\left(d^{+}, c^{+}\right)+g\left(d_{i}^{-}, c_{i}^{-}\right)+0.5\right)
\end{aligned}
$$

Similarly, for a question that requires choosing the most contradictive option, we will have an incorrect option $d^{-}$and several correct options $d_{1}^{+}, d_{2}^{+}, \ldots, d_{k}^{+}$, paired with their retrieved evidence chains $c^{-}, c_{1}^{+}, c_{2}^{+}, \ldots, c_{k}^{+}$, the loss will be:

$$
\begin{aligned}
& L\left(d^{-}, d_{1}^{+}, d_{2}^{+}, \ldots, d_{k}^{+}\right)= \\
& \sum_{i=1}^{k} \max \left(0,-g\left(d_{i}^{+}, c_{i}^{+}\right)+g\left(d^{-}, c^{-}\right)+0.5\right)
\end{aligned}
$$

During inference, we select the option with the highest score as the answer for a question requiring choosing the option that is the most consistent to the passage. Similarly, we select the option with the lowest score for a question asking for the option that contradicts most to the passage.

\subsection{Implementation Details}

For the iterative extractor, we use jieba ${ }^{5}$ to perform Chinese word segmentation. We use pre-trained word vectors (Qiu et al., 2018) to perform unsupervised iterative extraction with our query updating strategies. The $\lambda$ in Eq. 4 is set to 1 . Since $83 \%$ of

\footnotetext{
${ }^{5}$ https://github.com/fxsjy/jieba
}

the options with multiple evidence pieces require two evidence sentences, we set the maximum number of iterations to 2 . Considering that maintaining all evidence chains during iterative extraction has exponential complexity to the steps, we use beam search, where only top 2 evidence sentences remain in each step.

We use Sentence-BERT (Reimers and Gurevych, $2019,2020)$ to measure the relevance between the query and the evidence chains in the adaptive integrator. For the pairwise option competition, we use Chinese RoBERTa-wwm-ext-Large (Cui et al., 2019) with Transformers toolkit (Wolf et al., 2020). We first fine-tune our model on OCNLI (Hu et al., 2020), a Chinese natural language inference dataset before fine-tuning on VGaokao, which has 8 epochs, with maximum input length 256 , batch size 64 , and learning rate $2 \mathrm{e}-5$.

\section{Experiments}

We conduct experiments on our proposed VGaokao dataset and compare our ExtractIntegrate-Compete approach with several baselines.

RoBERTa-Large-Chunk (Liu et al., 2019) is an end-to-end method without explicit evidence retrieval. This model splits the long passages into fix-length chunks of 200 tokens. Candidate answers are obtained from each chunk using existing MRC models, which are further aggregated over all chunks. We use the pre-trained Chinese RoBERTawwm-ext-Large (Cui et al., 2019).

BM25 (Robertson and Zaragoza, 2009) is a bagof-words retrieval method, which uses sparse features to retrieve evidence sentences. We use the version implemented by Pyserini ${ }^{6}$.

Sent-BERT (Reimers and Gurevych, 2019) uses BERT to obtain contextualized dense representations for the texts and retrieve evidence sentences via cosine similarity.

BeamDR (Xiong et al., 2021; Zhao et al., 2021) is an iterative evidence selection technique with beam search and dense retrieval. It updates the query by appending the newly-extracted evidence in each iteration.

The BM25, Sent-BERT, and BeamDR are evidence extraction methods, which are combined with our proposed pair-wise option competition method to obtain the final question-level results. For BM25 and Sent-BERT, which cannot address the problem of multiple evidence, we report their

\footnotetext{
${ }^{6}$ https://github.com/castorini/pyserini
} 


\begin{tabular}{l|ccc|c}
\hline & \multicolumn{3}{|c|}{ Evidence Metrics } & QA Metrics \\
\cline { 2 - 5 } & $\mathbf{P}$ & $\mathbf{R}$ & $\mathbf{F 1}$ & Acc. \\
\hline RoBERTa-L-Chunk & - & - & - & 41.9 \\
\hline BM25 Top 1 & $\mathbf{9 3 . 0}$ & 71.1 & 77.9 & 47.6 \\
BM25 Top 2 & 64.5 & $\mathbf{8 7 . 7}$ & 71.6 & 48.4 \\
Sent-BERT Top 1 & 87.0 & 67.3 & 73.4 & 48.4 \\
Sent-BERT Top 2 & 55.5 & 79.1 & 62.8 & 48.9 \\
\hline BeamDR & 52.5 & 74.0 & 59.2 & 48.9 \\
\hline Hard Masking & 82.5 & 79.5 & $\mathbf{7 9 . 2}$ & 49.3 \\
Soft Masking & 82.5 & 77.5 & 78.0 & $\mathbf{4 9 . 6}$ \\
\hline
\end{tabular}

Table 3: Performance of baselines and our methods on VGaokao (\%). RoBERTa-L-Chunk is short for RoBERTa-Large-Chunk.

experiment results with top 1 or top 2 evidence sentences selected. To make fair comparison, we do not intensively tune any models, including ours. Specifically, we first search hyperparameters for the BM25 model, and apply the same hyperparameters to other baselines and our method.

We use three metrics to evaluate evidence quality on the subset annotated with golden evidence sentences: precision $(\mathbf{P})$, recall $(\mathbf{R})$, and F1 (F1). The accuracy of predicted answers (Acc.) is used to evaluate the performance on the question level.

\subsection{Main Results}

As we can see in Table 3, our Extract-IntegrateCompete approach outperforms the end-to-end baseline and other evidence retrieval methods in both evidence quality and answer prediction accuracy. We analyze the results by comparing 1) passage chunking and evidence selection, 2) one-off evidence selection and iterative evidence selection, and 3) different query updating strategies.

\section{Passage Chunking vs. Evidence Selection} RoBERTa-Large-Chunk simply chunks passages into pieces, which is a common practice for end-to-end MRC models (Devlin et al., 2019; Kwiatkowski et al., 2019). It obtains a questionlevel accuracy of $41.9 \%$, lagging behind the methods with additional evidence extraction step by at least $5.7 \%$. We think it is because the evidence sentences of a statement may appear in different chunks. Without evidence selection, models have to split the long article, which results in incomplete evidence for training and harm the performance. Even such simple methods as BM25 outperform the chunking method thanks to the retrieved evidence that is more relevant to the statement. Moreover, by introducing evidence selection, we can have a small-sized but focused supportive candidates to be feed the MRC model, greatly reducing required computational resources. These results illustrate the necessity of selecting evidence pieces from the long articles before performing verification.

One-off Selection vs. Iterative Selection In Table 3, our methods, with either hard masking or soft masking, outperform one-off evidence selection methods, BM25 and Sent-BERT settings, by $2 \sim 16 \%$ in the evidence extraction F1.

Specifically, by selecting the top 1 evidence sentence, these methods ensure a high precision but fail to provide sufficient evidence to the statements that require more evidence sentences. For example, in the first case of Table 4, the top 1 evidence sentence selected by S-BERT only covers the second half of the statement while our method with soft masking strategy succeeds in retrieving the complete evidence chain with iterative extraction.

On the other hand, by selecting top 2 evidence sentences, Sent-BERT and BM25 achieve a high recall but may introduce irrelevant evidence to the statements requiring fewer evidence sentences. For the second case in Table 4, using the top 2 evidence sentences will introduce a noisy sentence, while our method correctly determines that the statement only needs one evidence sentence with the adaptive evidence integrator.

Our evidence selection module achieves a balance between the precision and recall of the retrieved evidence pieces, obtaining the highest F1 scores among all methods. By iteratively extracting possible partial evidence, our methods ensure a relatively high recall of over $80 \%$; by adaptively integrating truly complementary evidence pieces, our methods lead to a relatively high precision of nearly $80 \%$. Therefore, our methods with iterative extraction and adaptive integration achieve the highest F1 scores among competitive counterparts. Besides, the higher evidence performance also results in better QA performance, with an improvement of more than $0.7 \%$. This indicates that carefully retrieved evidence produces more reliable evidence chains for the verification module, improving the explainability of our methods.

Query Updating Strategy Our query updating strategies, hard masking and soft masking, outperform BeamDR by approximately $20 \%$ in evidence retrieval F1. After each iteration, BeamDR appends 
Statement: 互联网颠覆传统制造业的现象在发达国家已出现；国内互联网巨头主动涉足传统制造业，互联网去工 业化初现端倪。

The phenomenon of the Internet subverting traditional manufacturing has already appeared in developed countries; domestic Internet giants have taken the initiative to set foot in traditional manufacturing, and the de-industrialization of the Internet has begun to take shape.

S-BERT Top 1 Evidence: (2) Soft Masking Evidence: (1)(2) Golden Evidence: (1)(2)

(1)互联网的去工具化从百度、腾讯等互联网巨头纷纷主动涉足传统制造业已经初现端倪。

The de-instrumentation of the Internet has begun to emerge from Internet giants such as Baidu and Tencent, which take the initiative to set foot in traditional manufacturing.

(2)而互联网对传统制造业带来的颠覆在发达国家也已出现。

The disruption of the traditional manufacturing industry brought by the Internet has also appeared in developed countries.

Statement: 美国某款新能源汽车生产者运用了物联网概念，取消了 $4 \mathrm{~S}$ 店的商业模式，自己销售产品并提供保养、 维修等各项服务。

A new-energy vehicle manufacturer in the United States used the concept of the Internet of Things, abandoned the business model of the 4S shop, sold its own products and provided various services such as maintenance and repair.

S-BERT Top 2 Evidence: (1) (2) Soft Masking Evidence: (1) Golden Evidence: (1)

(1)美国的某款新能源汽车, 由于运用了物联网概念, 已经取消了传统的4S店商业模式, 不仅销售不需要, 甚至保 养、维修也不再需要 $4 \mathrm{~S}$ 店

A new energy vehicle in the United States, due to the use of the Internet of Things concept, has abandoned the traditional 4S shop business model. Not only does it not need to be sold, but even maintenance and repairs do not require 4S shops.

(2)在工业4.0阶段，互联网已经不再是传统意义上的信息网络，更是物质、能量和信息互相交融的物联网，传递的 也不仅是传统意义上的信息，还可以包括物质和能量的信息。

In the 4.0 stage of industry, the Internet is no longer an information network in the traditional sense. It is an Internet of Things that integrates material, energy and information. It transmits not only information in the traditional sense, but also information about matter and energy.

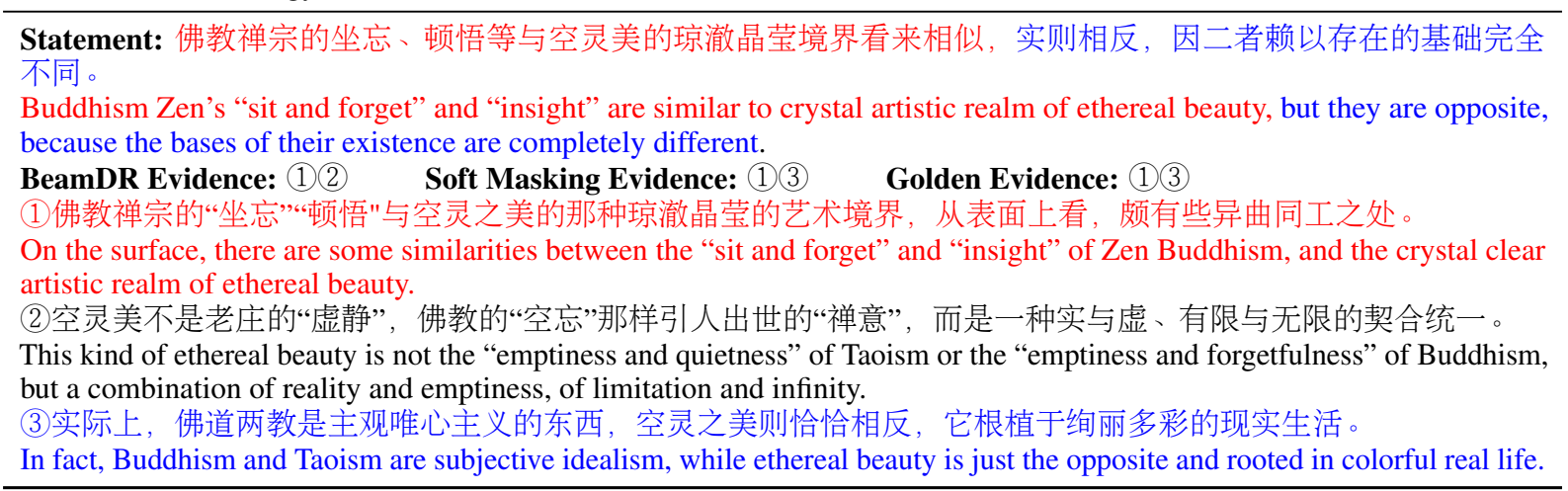

Table 4: Case study of the evidence retrieved by different methods. Golden evidence and its corresponding part in the statement are marked with the same color (red or blue).

the extracted evidence sentence to the query for following iterations. This strategy may put more emphasis on the overlap between retrieved evidence and queries, while the unmatched parts are likely to receive less attention. This tendency prevents the model from gathering complementary evidence sentences. By contrast, our query updating methods highlight the tokens in the query whose corresponding evidence is still missing, thus, being more likely to retrieve complementary evidence pieces.

For the third case of Table 4, the second evidence sentence returned by BeamDR shares the same topic with the first one, but irrelevant to the given statement. On the contrary, the second evidence sentence returned by our method is a complementary one that supports the second half of the statement. A possible reason is that our soft masking strategy can highlight the phrases such as opposite, basis of existence that are absent in the first retrieved evidence, pushing the retriever to collect complementary evidence.

Our two masking strategies, hard masking and soft masking, perform comparably on both metrics. The hard masking is easy to implement and requires little computation when updating queries; the soft masking can to some extent maintain the information in the matched tokens, which may be useful in the next step evidence retrieval. To summarize, our query updating strategies can make the retrieval target of each iteration more focused and boost the performance in both evidence extraction and the final answer selection. 


\begin{tabular}{l|l|l}
\hline Methods & Evi. F1 & Qu. Acc. \\
\hline Soft Masking & 78.0 & 49.6 \\
\hline w/o Iterative Extraction & $71.7(-6.3)$ & $47.7(-1.9)$ \\
w/o Adaptive Integration & $62.9(-15.1)$ & $47.4(-2.2)$ \\
w/o Pairwise Competition & - & $48.7(-0.9)$ \\
\hline
\end{tabular}

Table 5: Ablation study of our soft masking method on VGaokao.

\subsection{Ablation Study}

To reveal the effectiveness of each module in our approach, we conduct experiments with three ablated settings with soft masking for the iterative extractor. As we can see in Table 5, After we remove each of the three modules, the performance drops substantially in evidence F1 and questionlevel accuracy, demonstrating the effectiveness of each part in our approach.

Specifically, after removing the iterative extraction module, we can observe a decrease of $6.3 \%$ in the evidence $\mathrm{F} 1$ and a decrease of $1.9 \%$ in the question-level accuracy, because one-off evidence extraction fails to gather sufficient complementary evidence pieces. Thus the option competition module is unlikely to make a thoughtful decision based on an incomplete evidence chain.

When we remove the adaptive integration module, the evidence F1 drops by $15.1 \%$ and the question-level test accuracy drops by $2.2 \%$. This may result from irrelevant evidence pieces introduced in the later iterations of evidence extraction. The adaptive integration module can screen out this kind of noisy information by evaluating the associativity between extracted evidence, i.e., the property that evidence pieces are logically connected to cover the information in the query.

Without the pairwise option competition, the question-level accuracy decreases by $0.9 \%$. This indicates that pairwise option competition is superior to independent treatment of each option, because pairwise competition can push the model to capture the subtle difference between options.

\section{Related Works}

Several previous datasets are constructed from different subjects of Gaokao, including Geography (Huang et al., 2019), English (Lai et al., 2017; Sun et al., 2019), and History (Guo et al., 2017). Different from these efforts, we focus on Chinese subject to introduce native-speaker level reading comprehension challenges.
Evidence extraction based methods have been studied in MRC (Talmor and Berant, 2018; Perez et al., 2020) and fact verification (Thorne et al., 2018). In this work, we introduce evidence extraction to our method for solving the long article challenge in VGaokao.

Iterative evidence extraction can be seen as a sort of question decomposition method, a technique widely used in QA tasks with complex questions (Talmor and Berant, 2018; Perez et al., 2020). However, in VGaokao, the queries may interweave by implicit semantic relationship, so that models could not explicitly separate the queries into independent sub-queries. We thus adopt an iteative extractor with an adaptive integrator to decompose queries in an implicit way.

Another stream of works adopt an iterative framework by updating the queries. Qi et al. (2019) iteratively generate new queries by selecting a span from the question and retrieved evidence while Xu et al. (2019); Xiong et al. (2021); Zhao et al. (2021) directly append retrieved evidence to the query. Compared with these works, we introduce two novel query updating techniques, hard masking and soft masking, together with an evidence integration module to avoid too much overlap between evidence and to dynamically determine the number of required evidence sentences.

\section{Conclusion}

In this paper, we present a novel verification style reading comprehension dataset named VGaokao from the Chinese Language tests of Gaokao for Chinese native speakers, which embed multiple advanced language understanding skills. To address the challenges in VGaokao, we propose a new Extract-Integrate-Compare approach for complementary evidence retrieval/integration and option discrimination. Experiments show that our approach outperforms several strong baselines, with additional merits of efficiency and explainability. We believe VGaokao is a challenging test-bed for natural language understanding in Chinese and encourage further research in verification style reading comprehensionn.

\section{Acknowledgements}

We thank the anonymous reviewers for the helpful comments and suggestions. This work is supported by Hi-Tech R\&D Program of China (No.2018YFB1005100). 


\section{References}

Yiming Cui, Wanxiang Che, Ting Liu, Bing Qin, Ziqing Yang, Shijin Wang, and Guoping Hu. 2019. Pre-training with whole word masking for chinese bert. arXiv preprint arXiv:1906.08101.

Jacob Devlin, Ming-Wei Chang, Kenton Lee, and Kristina Toutanova. 2019. Bert: Pre-training of deep bidirectional transformers for language understanding. In Proceedings of the 2019 Conference of the North American Chapter of the Association for Computational Linguistics: Human Language Technologies, Volume 1 (Long and Short Papers), pages 4171-4186.

Shangmin Guo, Xiangrong Zeng, Shizhu He, Kang Liu, and Jun Zhao. 2017. Which is the effective way for gaokao: information retrieval or neural networks? In Proceedings of the 15th Conference of the European Chapter of the Association for Computational Linguistics: Volume 1, Long Papers, pages 111-120.

Hai Hu, Kyle Richardson, Liang Xu, Lu Li, Sandra Kübler, and Lawrence S Moss. 2020. Ocnli: Original chinese natural language inference. In Proceedings of the 2020 Conference on Empirical Methods in Natural Language Processing: Findings, pages 3512-3526.

Zixian Huang, Yulin Shen, Xiao Li, Gong Cheng, Lin Zhou, Xinyu Dai, Yuzhong Qu, et al. 2019. Geosqa: A benchmark for scenario-based question answering in the geography domain at high school level. In Proceedings of the 2019 Conference on Empirical Methods in Natural Language Processing and the 9th International Joint Conference on Natural Language Processing (EMNLP-IJCNLP), pages 58695874.

Tom Kwiatkowski, Jennimaria Palomaki, Olivia Redfield, Michael Collins, Ankur Parikh, Chris Alberti, Danielle Epstein, Illia Polosukhin, Jacob Devlin, Kenton Lee, et al. 2019. Natural questions: A benchmark for question answering research. Transactions of the Association for Computational Linguistics, 7:453-466.

Guokun Lai, Qizhe Xie, Hanxiao Liu, Yiming Yang, and Eduard Hovy. 2017. Race: Large-scale reading comprehension dataset from examinations. In Proceedings of the 2017 Conference on Empirical Methods in Natural Language Processing, pages 785794.

Yinhan Liu, Myle Ott, Naman Goyal, Jingfei Du, Mandar Joshi, Danqi Chen, Omer Levy, Mike Lewis, Luke Zettlemoyer, and Veselin Stoyanov. 2019. Roberta: A robustly optimized bert pretraining approach. arXiv preprint arXiv:1907.11692.

Ethan Perez, Patrick Lewis, Wen-tau Yih, Kyunghyun Cho, and Douwe Kiela. 2020. Unsupervised question decomposition for question answering. In Proceedings of the 2020 Conference on Empirical
Methods in Natural Language Processing (EMNLP), pages 8864-8880.

Peng Qi, Xiaowen Lin, Leo Mehr, Zijian Wang, and Christopher D Manning. 2019. Answering complex open-domain questions through iterative query generation. In Proceedings of the 2019 Conference on Empirical Methods in Natural Language Processing and the 9th International Joint Conference on Natural Language Processing (EMNLP-IJCNLP), pages 2590-2602.

Yuanyuan Qiu, Hongzheng Li, Shen Li, Yingdi Jiang, Renfen Hu, and Lijiao Yang. 2018. Revisiting correlations between intrinsic and extrinsic evaluations of word embeddings. In Chinese Computational Linguistics and Natural Language Processing Based on Naturally Annotated Big Data, pages 209-221. Springer.

Pranav Rajpurkar, Jian Zhang, Konstantin Lopyrev, and Percy Liang. 2016. Squad: 100,000+ questions for machine comprehension of text. In Proceedings of the 2016 Conference on Empirical Methods in Natural Language Processing, pages 2383-2392.

Nils Reimers and Iryna Gurevych. 2019. Sentencebert: Sentence embeddings using siamese bertnetworks. In Proceedings of the 2019 Conference on Empirical Methods in Natural Language Processing and the 9th International Joint Conference on Natural Language Processing (EMNLP-IJCNLP), pages 3973-3983.

Nils Reimers and Iryna Gurevych. 2020. Making monolingual sentence embeddings multilingual using knowledge distillation. In Proceedings of the 2020 Conference on Empirical Methods in Natural Language Processing (EMNLP), pages 4512-4525.

Stephen Robertson and Hugo Zaragoza. 2009. The probabilistic relevance framework: BM25 and beyond. Now Publishers Inc.

Kai Sun, Dian Yu, Jianshu Chen, Dong Yu, Yejin Choi, and Claire Cardie. 2019. Dream: A challenge data set and models for dialogue-based reading comprehension. Transactions of the Association for Computational Linguistics, 7:217-231.

Kai Sun, Dian Yu, Dong Yu, and Claire Cardie. 2020. Investigating prior knowledge for challenging chinese machine reading comprehension. Transactions of the Association for Computational Linguistics, 8:141-155.

Alon Talmor and Jonathan Berant. 2018. The web as a knowledge-base for answering complex questions. In Proceedings of the 2018 Conference of the North American Chapter of the Association for Computational Linguistics: Human Language Technologies, Volume 1 (Long Papers), pages 641-651.

James Thorne, Andreas Vlachos, Christos Christodoulopoulos, and Arpit Mittal. 2018 Fever: a large-scale dataset for fact extraction and 
verification. In Proceedings of the 2018 Conference of the North American Chapter of the Association for Computational Linguistics: Human Language Technologies, Volume 1 (Long Papers), pages 809-819.

Thomas Wolf, Julien Chaumond, Lysandre Debut, Victor Sanh, Clement Delangue, Anthony Moi, Pierric Cistac, Morgan Funtowicz, Joe Davison, Sam Shleifer, et al. 2020. Transformers: State-of-theart natural language processing. In Proceedings of the 2020 Conference on Empirical Methods in Natural Language Processing: System Demonstrations, pages $38-45$.

Wenhan Xiong, Xiang Lorraine Li, Srini Iyer, Jingfei Du, Patrick Lewis, William Yang Wang, Yashar Mehdad, Wen-tau Yih, Sebastian Riedel, Douwe Kiela, et al. 2021. Answering complex open-domain questions with multi-hop dense retrieval. Proceedings of the International Conference on Learning Representations.

Kun Xu, Yuxuan Lai, Yansong Feng, and Zhiguo Wang. 2019. Enhancing key-value memory neural networks for knowledge based question answering. In Proceedings of the 2019 Conference of the North American Chapter of the Association for Computational Linguistics: Human Language Technologies, Volume 1 (Long and Short Papers), pages 29372947.

Zhilin Yang, Peng Qi, Saizheng Zhang, Yoshua Bengio, William Cohen, Ruslan Salakhutdinov, and Christopher D Manning. 2018. Hotpotqa: A dataset for diverse, explainable multi-hop question answering. In Proceedings of the 2018 Conference on Empirical Methods in Natural Language Processing, pages 2369-2380.

Sheng Zhang, Xiaodong Liu, Jingjing Liu, Jianfeng Gao, Kevin Duh, and Benjamin Van Durme. 2018. Record: Bridging the gap between human and machine commonsense reading comprehension. arXiv preprint arXiv:1810.12885.

Chen Zhao, Chenyan Xiong, Jordan Boyd-Graber, and Hal Daumé III. 2021. Multi-step reasoning over unstructured text with beam dense retrieval. In Proceedings of the 2021 Conference of the North American Chapter of the Association for Computational Linguistics: Human Language Technologies, pages 4635-4641.

Chujie Zheng, Minlie Huang, and Aixin Sun. 2019. ChID: A large-scale Chinese IDiom dataset for cloze test. In Proceedings of the 57th Annual Meeting of the Association for Computational Linguistics, pages 778-787. 